\title{
La crisis del sistema de salud colombiano: una aproximación desde la legitimidad y la regulación*
}

\section{The Crisis of the Colombian Health System: An Approach from Legitimacy and Regulation}

\section{A crise do sistema de saúde colombiano: aproximação desde a legitimidade e a regulamentação}

Fecha de recepción: 12-12 2016 Fecha de aceptación: 22-03-2017 Disponible en línea: 30-05-2017 doi: doi:10.11144/Javeriana.rgps16-32.cssc

Cómo citar este artículo:

Suárez-Rozo LF, Puerto-García S, Rodríguez-Moreno LM, Ramírez-Moreno. La crisis del sistema de salud colombiano: una aproximación desde la legitimidad y la regulación. Rev Gerenc Polít Salud. 2017; 16 (32): 34-50. https://doi.org/10.11144/Javeriana.rgps16-32.cssc

\author{
Luisa Fernanda Suárez-Rozo** \\ Stephanie Puerto-García*** \\ Lina María Rodríguez-Moreno**** \\ Jaime Ramírez-Moreno*****
}

Artículo de investigación asociado al proyecto Factores condicionantes de los mercados, agentes e instituciones en el sector salud en relación con la regulación y la inspección, vigilancia y control en el Sistema General de Seguridad Social en Salud, el cual inició el 4 de octubre del 2013 y finalizó el 30 de abril del 2015. El proyecto fue financiado por Colciencias en el marco de un programa más amplio de investigación llamado Desarrollo y evaluación de un modelo integrado para la inspección, vigilancia y control del acceso oportuno, el uso adecuado y la calidad en la prestación de servicios en el SGSSS colombiano.

** Autora principal, Politóloga e historiadora, MSc en Estudios Ambientales y Desarrollo Sostenible en Lund University, profesora instructora en el Instituto de Salud Pública de la Pontificia Universidad Javeriana. Dirección

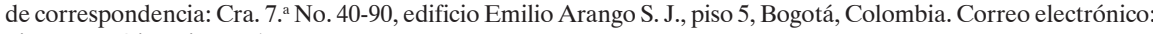
uisasuarez@javeriana.edu.co

*** Economista, estudiante de la Maestría en Economía en la Pontificia Universidad Javeriana, profesora instructora en el Instituto de Salud Pública de la Pontificia Universidad Javeriana, Correo electrónico: puertos@javeriana. edu.co

**** Psicóloga, magíster en Estudios Interdisciplinarios sobre Desarrollo de la Universidad de Los Andes. Consultora del Instituto de Salud Pública de la Pontificia Universidad Javeriana. Correo electrónico: linamariarodriguezm@ gmail.com

***** Economista, Ph. D. en Economía y Gestión de la Salud de la Universidad Politécnica de Valencia, España. Profesor asociado en el Instituto de Salud Pública de la Pontificia Universidad Javeriana. Correo electrónico: ramirez. jaime@javeriana.edu.co 


\section{Resumen}

El objetivo fue determinar cuáles son las percepciones de distintos actores del sistema de salud colombiano sobre los problemas del sistema que están detrás de la crisis. Se realizó un estudio cualitativo a partir de entrevistas semiestructuradas y en profundidad con cuarenta agentes del sector salud, en Bogotá, Cali, Ibagué, Espinal y Calima-Darién. Los resultados mostraron que la corrupción, el incumplimiento de normas y los conflictos entre los intereses de diversos actores del sistema debilitan la legitimidad de este último, atomizan la cooperación y cohesión de los actores y fortalecen el interés particular. La polución normativa, la inconsistencia temporal de las normas y la información incompleta generan incertidumbre, confusión y desintegración en el interior del sistema, con lo cual contribuyen al desenvolvimiento de una crisis regulatoria con respecto a la cual se hace urgente recuperar espacios de toma de decisiones colectivas y concertadas que promuevan el diálogo y la superación de los intereses rentistas individualistas.

Palabras clave: sistemas de salud; aseguradoras; prestación de atención de salud; accesibilidad a los servicios de salud; derecho a la salud; legislación sanitaria

\section{Abstract}

The objective was to determine the perceptions of different actors of the Colombian health system regarding the problems of the system behind the crisis. A qualitative study was carried out on the basis of semi-structured and in-depth interviews with forty health sector agents in Bogotá, Cali, Ibagué, Espinal, and Calima-Darién. The results showed that corruption, breach of regulations, and conflicts of interests between various actors in the system weaken the legitimacy of the system, shatter the cooperation and cohesion of the actors, and strengthen private interests. Regulatory pollution, a temporary inconsistency of regulations, and incomplete information generate uncertainty, confusion, and disintegration within the system. This, in turn, contributes to the development of a regulatory crisis regarding which it is urgent to recover collective and concerted decision-making spaces to promote dialogue and the overcoming of rentier individualistic interests.

Keywords: health systems; insurance carriers; delivery of health care; health services accessibility; right to health; health legislation

\section{Resumo}

O objetivo foi determinar quais as percepções de diferentes atores do sistema de saúde colombiano sobre os problemas do sistema por trás da crise. Estudo qualitativo a partir de entrevistas semiestruturadas e em profundidade a quarenta agentes do setor saúde, em Bogotá, Cali, Ibagué, Espinal e Calima-Darién foi realizado. Os resultados mostraram que a corrupção, o descumprimento de regras e os conflitos entre os interesses de diversos atores do sistema enfraquecem a legitimidade deste último, atomizam a cooperação e coesão dos atores e fortalecem o interesse particular. A poluição normativa, a inconsistência temporal das regras e a informação incompleta criam incerteza, confusão e desintegração no interior do sistema, com o qual contribuem para o desenvolvimento de uma crise regulatória com respeito à qual resulta urgente recuperar espaços de tomada de decisões coletivas e concertadas a promover o diálogo e a superação dos interesses rentistas individualistas.

Palavras chave: sistemas de saúde; asseguradoras; prestação de cuidados de saúde; acessibilidade ao serviço de saúde; direito à saúde; legislação sanitária 


\section{Introducción}

Colombia inicia en la década de los años noventa un proceso denominado la revolución pacífica, que pretendía desarrollar la Constitución del 91 para la construcción del Estado social de derecho y mejorar la posición del país en el mercado internacional, a través de un proceso de apertura económica y un programa de modernización del Estado. Entre los instrumentos de dicha transformación, se desarrollaron leyes y programas sociales basados en la teoría del capital humano, tendientes a transformar el sistema pensional, el mercado laboral, el sistema de protección de riesgos laborales y el sistema de salud, a efectos de lo cual se creó el Sistema Integral de Seguridad Social.

Las bases del programa de modernización tenían como punto central el cambio de las funciones sociales y económicas del Estado, pasando de un Estado proveedor de servicios a uno regulador, en la medida en que los nuevos sistemas sociales combinaban la regulación pública con la acción de los mercados y los agentes privados, en sistemas generales de seguridad social como el sistema de salud. Las reformas en salud se desarrollaron conservando el sistema corporativo del seguro social tradicional, con la existencia de un sistema segmentado y paralelo de seguros subsidiados para la población pobre y vulnerable.

El Sistema General de Seguridad Social en Salud (SGSSS) ${ }^{1}$ está conformado por un conjunto de instituciones y mercados que configuran las relaciones entre el sistema y los ciudadanos, a través de la afiliación obligatoria, la cotización obligatoria y la cobertura de riesgos amparadas en planes obligatorios de salud segmentados en regímenes especiales,

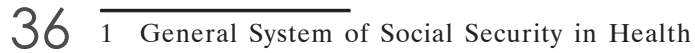
(GSSSH). régimen contributivo, régimen subsidiado y un sistema de atención mediante la oferta de hospitales públicos para la población no afiliada.

Se ha documentado una variedad de situaciones problemáticas inherentes al sistema, las cuales incluyen la distribución inequitativa de recursos humanos en las regiones, servicios muy demandados por la población y excluidos del Plan Obligatorio de Salud $(\mathrm{POS})^{2}$, diferencias en la cobertura entre el plan ofrecido al régimen contributivo y el plan ofrecido al régimen subsidiado, conflictos entre prestadores de servicios de salud y aseguradoras en salud, baja capacidad institucional en territorios, escasa autonomía de prestadores de servicios de salud y crisis de hospitales públicos, entre otras $(2)^{3}$. En ese contexto, los ciudadanos han apelado de forma recurrente a la tutela, como medio para amparar su derecho a la salud $(2,5,6)$. Producto de este fenómeno, la

2 Mandatory Health Plan (MHP). El POS es el paquete de medicamentos, procedimientos y servicios de salud cubiertos para los afiliados a los distintos regímenes de salud. A través de la Sentencia T-760 del 2008, la Corte Constitucional hizo un llamado al Ministerio de Salud para unificar los POS de los regímenes subsidiado y contributivo, que anteriormente estaban compuestos por listas de medicamentos, procedimientos y servicios diferentes. La unificación se ha venido haciendo, pero aún quedan elementos por uniformizar. El POS se extiende a las áreas de recuperación de la salud, prevención de la enfermedad y prestaciones económicas por enfermedad, accidentes o maternidad (1).

3 En el Sistema de Salud colombiano los prestadores de servicios de salud son conocidos con el nombre de Instituciones Prestadoras de Servicios (IPS), y se definen como todas las instituciones, bien sean públicas, privadas o de economía mixta, autorizadas para prestar de forma parcial o total servicios de salud. A su vez, las IPS públicas se conocen como Empresas Sociales del Estado (ESE), las cuales constituyen entidades públicas de carácter especial, con personería jurídica, autonomía administrativa, patrimonio propio y son descentralizadas (3). Por su parte, las Entidades Promotoras de Salud (EPS) son el equivalente a las aseguradoras en el sistema y como tal son responsables de promover la afiliación de los grupos de población que actualmente no están cubiertos por la seguridad social; organizar los mecanismos a través de los cuales los afiliados 
Corte Constitucional (CC) $)^{4}$ elevó el derecho a la salud a derecho fundamental principal, ya que el Estado no había tomado suficientes medidas de intervención y regulación para corregir dichas inequidades en el sistema. Como mecanismo para hacer cumplir el derecho a la salud, la CC dictó la Sentencia T-760, donde obliga al Estado a unificar los planes de beneficios entre los regímenes y a realizar un programa de reformas para garantizar el goce efectivo del derecho a la salud (GED) ${ }^{5}$. La severidad de esta situación llevó al Gobierno nacional a declarar la emergencia social en el año 2009 (Decreto 4975), buscando con ello solucionar los serios problemas financieros que amenazaban la estabilidad del sistema.

Debido a esta acción pública de carácter regulatorio realizada por la $\mathrm{CC}$, se inician procesos legislativos para llevar a cabo las reformas al sistema de salud en términos de la ley estatutaria y la denominada ley ordinaria. El proceso político logra aprobar la ley estatutaria, corregida por la CC, pero la ley ordinaria no pasa. Lo anterior crea una situación particular en el sistema de salud colombiano, donde existe una definición amplia del derecho a la salud, de carácter constitucional, pero sin medios y recursos para conseguir los objetivos, lo cual origina grandes insatisfacciones entre los usuarios del sistema, hasta el punto de que se generan dos procesos: primero, pérdida de legitimidad del sistema frente a los ciudadanos, quienes

y sus familias acceden a los servicios de salud en el territorio nacional; y captar los aportes de los afiliados al SGSSS actuando como delegatarias del Fondo de Solidaridad y Garantía (Fosyga), entre otros (4).

En este artículo solo se utilizará el vocabulario nacional (IPS, ESE, EPS) en las citas textuales de las entrevistas, mientras que en el resto del texto se emplearán los términos más genéricos de prestadores de servicios, hospital público y aseguradoras en salud.

4 Constitutional Court (CC).

5 Effective enjoyment of the right to health (EERH). consideran que este empeora, pues no satisface las expectativas ni las necesidades de la población; y segundo, inestabilidad económica provocada por la crisis financiera de las aseguradoras, generalización de las deudas con los prestadores y falta de oportunidad en la atención a las personas.

Como la ley estatutaria y la ordinaria fueron debatidas públicamente por los diferentes agentes del sistema de salud, representan un espectro amplio y consensuado de las problemáticas del sistema. Sin embargo, la literatura científica no cuenta con un estudio detallado de las percepciones de los actores del sistema acerca de estos problemas, ni en lo relacionado con la calidad de la inspección, vigilancia y control(IVC) ${ }^{6}$ y la regulación que deben ejercer la Superintendencia Nacional de Salud(SNS ${ }^{7}$ y el Ministerio de Salud y Protección Social(MSPS) ${ }^{8}$, respectivamente. Para llenar estos vacíos de conocimiento, se consideró pertinente hacer una investigación cualitativa donde participaran diversos agentes que intervienen en el sistema, con el fin de construir un diagnóstico de la situación de crisis que atraviesa la salud, el cual permita proponer mejoras al sistema actual de IVC en salud, a escala territorial. La investigación, iniciada en el año 2013, examinó las experiencias y las percepciones de los agentes del sistema de salud en cuanto a: 1) los problemas del Sistema, 2) la calidad de la IVC, 3) la calidad de la regulación y 4) las expectativas respecto al futuro del sistema. Por la complejidad y la extensión de la información recopilada, este artículo presenta únicamente los resultados relacionados con los problemas del sistema. Investigar estas cuestiones es absolutamente relevante en el contexto actual, en el cual se ha aprobado la ley estatutaria, ley que reconoce el derecho a

\footnotetext{
6 Inspection, Surveillance and Control (ISC).

7 National Health Supervisory Agency (NHSA).

8 Ministry of Health and Social Protection (MHSP).
} 
la salud como un derecho fundamental, pero se ha rechazado la ley ordinaria que buscaba reglamentarla, en tanto que las medidas para llevarla a la práctica se han adoptado por la vía del decreto o el plan de desarrollo. Este artículo busca contribuir a la comprensión del contexto de crisis y a la identificación de herramientas para superarla, que vayan de la mano de la puesta en práctica de la ley estatutaria en salud.

Al explorar las percepciones sobre los problemas se buscaba responder a la pregunta por cuáles son los problemas que los agentes consideran importantes, y cómo los entienden. A través de las entrevistas y a la luz de los datos recogidos, se identificaron dos categorías de análisis propicias para dar cuenta de los problemas del sistema: 1) la legitimidad en el sistema de salud y 2) la crisis regulatoria generalizada.

La legitimidad en el sistema alude a la forma como la totalidad de los agentes, incluidos los reguladores, representan conceptualmente las manifestaciones y los hechos cotidianos que afectan los derechos de afiliación y uso de los beneficios del sistema, y que, dada su continuidad, terminan afectando la visión negativa o positiva que se tiene del sistema y la materialización de los derechos en salud. Al aproximarnos a la legitimidad en el sistema, se quería responder a las siguientes preguntas: 1) ¿de qué manera se evidencia la corrupción en el sistema y cómo la definen los entrevistados?, 2) ¿cómo se entiende el incumplimiento en las interacciones en el interior del sistema?, y 3 ) ¿en qué consisten los conflictos que surgen entre los intereses de distintos agentes del sistema? $\mathrm{Al}$ referirnos a la crisis regulatoria generalizada, indagamos por cuáles son las fallas regulatorias que se identifican en el sistema y la forma en que los agentes las entienden, incluyendo fallas como los problemas de información incompleta y la inconsistencia temporal en las normas del sistema. Esta categoría también se preguntaba por la evaluación que hacen los participantes de los espacios de toma de decisiones regulatorias y políticas, examinando si los conciben como escenarios que deben ser puramente técnicos, de concertación o de consulta.

\section{Métodos}

\section{Participantes y muestra}

Como parte del enfoque cualitativo, la muestra se seleccionó intencionalmente mediante un muestreo por conveniencia que busca comprender el fenómeno examinado, eligiendo individuos y contextos desde los cuales se pueda aprender mucho sobre este (7). Con esto en mente, los participantes en las entrevistas fueron seleccionados por ser directivos que participan de la toma de decisiones sobre política y regulación o el sistema de IVC; por ser propietarios o directivos de aseguradoras o prestadoras y sus respectivos gremios; por su condición de jueces municipales o magistrados de la CC; o por su papel de usuarios. El amplio espectro de agentes permitió alcanzar una visión comprehensiva y detallada de la diversidad de percepciones y del sistema en su totalidad.

Para identificar a los agentes se elaboró un mapa de actores del sistema de salud, por subsectores (véase figura 1). La selección estuvo sujeta a la disponibilidad de los individuos y su deseo de participar en las entrevistas. En total se realizaron cuarenta entrevistas entre julio y octubre del 2014, y cada entrevistado firmó un consentimiento informado autorizando o negando la grabación de la entrevista y la inclusión de citas textuales en el informe final del proyecto. El número de entrevistados para cada grupo de agentes se muestra en la figura 1 . 


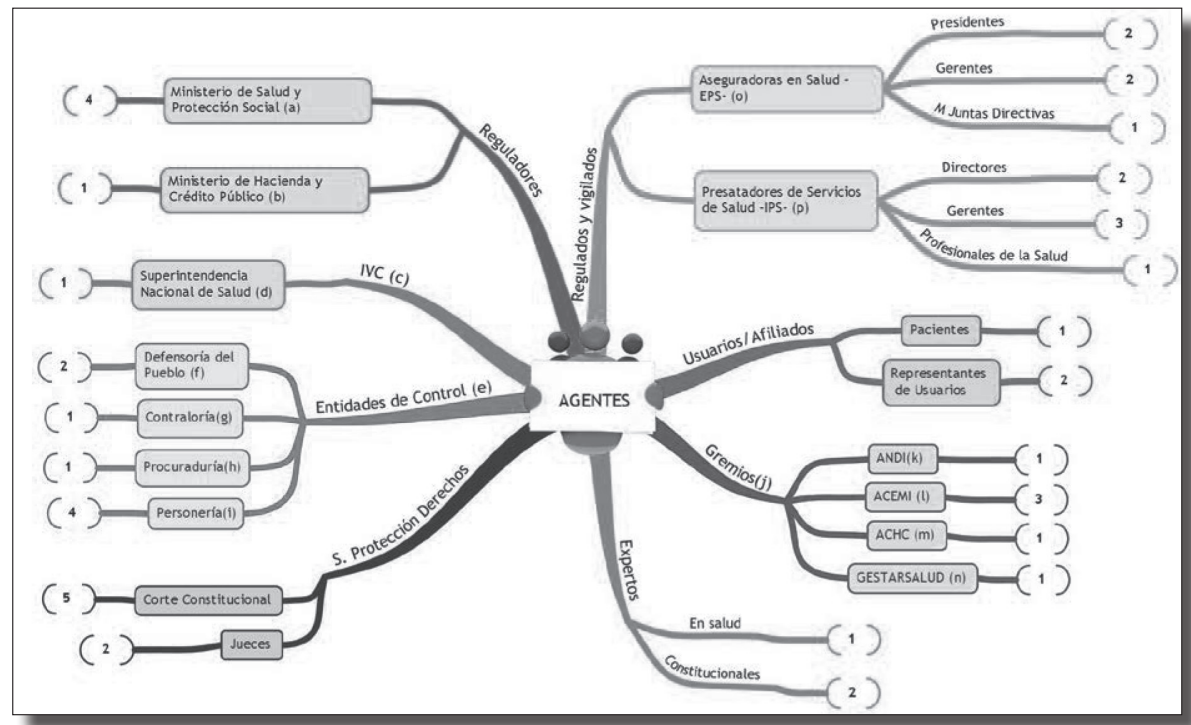

a Ministry of Health and Social Protection

b Ministry of Finance and Public Credit

c Inspection, Surveillance and Control

d National Health Supervisory Agency

e Oversight Organizations

f Ombudsman

g National Auditing Office

h Public Prosecutor's Office i Municipal Ombudsman

j Trade Unions

k National Colombian Association of Entrepreneurs

1 Colombian Association of Comprehensive Medicine Enterprises

m Colombian Association of Hospitals and Clinics

n Health Insurance Management Companies Association

o Health Insurance Carriers

p Healthcare Service Providers

Figura 1. NúMERo DE ENTREVISTAS ANALIZADAS POR GRUPOS DE AGENTES

Fuente: elaboración propia

Las entrevistas se aplicaron a actores de Bogotá y de los departamentos de Valle y Tolima. La selección de los municipios se hizo tomando como referencia el Índice de Tamaño Funcional, el cual clasifica los municipios del país de acuerdo con la magnitud de sus funciones urbanas. Esto con el objetivo de dar cuenta de las distintas perspectivas presentes en los territorios de diferentes niveles de magnitud en el país. Se tomó a Bogotá por representar un "centro metropolitano nacional" y también porque alberga el nivel central de gobierno. Cali fue seleccionada como "centro metropolitano regional”, Ibagué como "centro regional mayor", el Espinal como "centro subregional mayor" y Calima-Darién por su condición de "centro local" (8).

\section{Entrevistas en profundidad y semiestructuradas}

Para recoger los datos se aplicaron entrevistas semiestructuradas y en profundidad. Esta última permitió obtener un conocimiento detallado del objeto estudiado y capturar la complejidad de fenómenos sociales que, en apariencia, son sencillos (9). También facilitó el análisis temático de la información recopilada (10), lo cual permitió profundizar en una 
gran diversidad de temas y sus relaciones. Al incorporar la entrevista semiestructurada se contó con una guía de preguntas abiertas para utilizar con flexibilidad en cada situación y se pudo establecer una conversación entre entrevistado y entrevistador $(9,11)$. Dado el grado de detalle que alcanzan y la flexibilidad que las caracteriza, este tipo de entrevistas resultaron especialmente pertinentes para examinar a fondo las percepciones de los agentes.

Los resultados aquí analizados examinan el tema de los problemas del sistema, a través de las categorías y códigos que se muestran en la tabla 1. Luego de realizadas, las entrevistas fueron transcritas para su posterior codificación y análisis, utilizando el software NVivo 10.

TABla 1. Temas, CATEgorías y Códigos InVESTIGADOS

\begin{tabular}{|c|c|c|c|}
\hline Tema & Categoría & Código 1 & Código 2 \\
\hline \multirow{10}{*}{$\begin{array}{l}\text { Problemas } \\
\text { del sistema }\end{array}$} & \multirow{5}{*}{ Legitimidad } & Corrupción & \\
\hline & & Incumplimiento & \\
\hline & & \multirow{3}{*}{$\begin{array}{l}\text { Conflictos entre los inte- } \\
\text { reses de los agentes: lo } \\
\text { individual y lo colectivo }\end{array}$} & Conflictos aseguradoras-prestadores-usuarios \\
\hline & & & Conflictos con el Estado \\
\hline & & & Prima interés financiero sobre el GED \\
\hline & \multirow{5}{*}{$\begin{array}{l}\text { Crisis } \\
\text { regulatoria } \\
\text { generalizada }\end{array}$} & Fallas regulatorias & \\
\hline & & Información incompleta & \\
\hline & & Inconsistencia temporal & \\
\hline & & \multirow{2}{*}{$\begin{array}{l}\text { Instituciones y prácticas } \\
\text { regulatorias para la toma } \\
\text { de decisiones }\end{array}$} & \\
\hline & & & \\
\hline
\end{tabular}

Fuente: elaboración propia

\section{Análisis de los datos}

En el marco de este estudio se realizó un análisis temático de los datos recopilados. Este tipo de análisis es propicio para investigar las percepciones de los agentes entrevistados, dado que permite romper los textos de las entrevistas en conjuntos pequeños de contenido, para luego describirlos en detalle. Adicionalmente, facilita la identificación de patrones en los datos y de convergencias en las visiones y experiencias de los participantes del proyecto. El análisis siguió las etapas que componen el análisis temático $(12,13)$.

El proceso de codificación fue tanto deductivo como inductivo (14). Primero, se diseñó una tabla con códigos y categorías que surgieron de las preguntas de las guías de entrevista, previamente al proceso de codificación. Una vez finalizada la tabla, se procedió a codificar una entrevista en grupo, para probar la pertinencia de los códigos definidos deductivamente, y para tratar de unificar los criterios de codificación que guiarían a los distintos investigadores al momento de codificar de manera individual. Posteriormente, al individualizar la codificación, emergieron códigos nuevos que iban surgiendo en forma inductiva de los datos, a medida que cada investigador leía y se familiarizaba con los textos. Los investigadores discutieron los códigos emergentes para determinar si se aprobaban, o para cualificar su definición. A lo largo del proceso fue necesario revisar las entrevistas ya codificadas, para asegurar que los códigos emergentes se aplicaran a todos los datos. Luego de empezar a codificar 
también fue necesario redefinir y renombrar algunos códigos que se habían creado de forma deductiva, para que correspondieran efectivamente con los datos codificados.

La creación de categorías consiste en agregar bajo un nombre distintos códigos que guardan una relación (13). De la misma manera que los códigos, las categorías se definieron tanto deductiva como inductivamente, y cuando fue necesario se modificaron los nombres para representar mejor los datos (véase tabla 1 ).

Los temas son diferentes de las categorías, en la medida que no se limitan a describir el contenido de los datos de estas, sino que involucran cierto grado de interpretación de los datos, relacionado con el tema de investigación examinado (13). En palabras de Vaismoradi, Turunen y Bondas, el tema “[...] como mínimo describe y organiza información, y como máximo interpreta aspectos de un fenómeno" (14). En la misma dirección, varios autores han explicado que el tema recoge algo importante en los datos, que está relacionado con la pregunta de investigación y exhibe cierto patrón de respuesta o significado reflejado por los datos $(14,15)$. Para propósitos de este artículo se exponen solo los resultados relacionados con el tema de problemas del sistema de salud (véase tabla 1).

\section{Resultados}

Esta sección presenta los resultados de investigación en lo relativo a las categorías de legitimidad en el sistema de salud y crisis regulatoria generalizada, que hacen parte del tema más amplio de los problemas del sistema de salud colombiano.

\section{Legitimidad en el sistema de salud}

Esta categoría incluye los códigos referidos la corrupción, el incumplimiento y los conflictos entre los intereses de los agentes: lo individual y lo colectivo.

Corrupción. En lo que se refiere a la corrupción, esta se asocia, según varios agentes, a las prácticas indebidas en el manejo de los recursos por parte de las aseguradoras. Refiriéndose al caso de la aseguradora SaludCoop, un juez de Espinal comentó que "[...] el dinero de los usuarios [...] y de los empleadores [...] se está destinando a satisfacer intereses no de los usuarios, sino de índole privada" (Entrevista 21). En una misma línea, una funcionaria de un organismo de control y el director de un hospital privado en Bogotá consideraron que el problema de la salud no es un tema de recursos limitados, sino que se da por la desviación de recursos a manos de los corruptos. En palabras de uno de ellos, "los recursos deberían alcanzar [y] casi que ser suficientes para un sistema [...] eficiente. Yo creo, que más que recursos, el problema ha sido la plata que se pierde por corrupción, por ineficiencias [...]" (entrevistas 37 y 10). Por su parte, algunos gerentes de hospitales públicos de Cali y Calima sostuvieron que la corrupción se teje en la interacción entre actores públicos y privados, pero son estos últimos quienes la desencadenan (entrevistas 1 y 24).

Incumplimiento. Distintos agentes señalan la existencia de un amplio número de normas que regulan el sector, pero que no se cumplen. Están en el papel y, sin embargo, no se aplican en la práctica. En este sentido, el director de un hospital privado en la ciudad de Bogotá expresa que no es necesario "[...] hacer más decretos [...]", si estos no se cumplen, y agrega que "[...] todos saben que la posibilidad de que violar la norma les signifique algo es bajita, entonces uno necesita menos normas pero con menos impunidad [...]" (Entrevista 30). Esta situación de incumplimiento de normas, en las múltiples formas que puede asumir, y la condición de impunidad que constantemente 
se produce, son entendidas como factores que restan legitimidad al sistema, porque generan desorden en su interior y fallas en su operación, lo que puede producir una imagen negativa del sistema, a los ojos de los usuarios y de los otros agentes. Además, esa visión se incrementa en la medida que el derecho de los ciudadanos a la salud se ve afectado por el incumplimiento y la impunidad.

Conflictos entre los intereses de los agentes: lo individual y lo colectivo. Conflictos aseguradoras-prestadores-usuarios. Algunos agentes entrevistados coinciden en afirmar que los conflictos entre los intereses de los agentes del sistema están ligados a la obtención de lucro económico. Para el gerente de un hospital público en Calima,

Mientras uno, aquí como prestador, tiene la angustia del paciente en urgencias que necesita remitir porque su vida está en riesgo [...] al otro lado de la línea hay una persona entrenada por la EPS para bajar costos y no está pensando tanto en la vida del paciente sino en lo que le cuesta a la EPS, y ese es el mayor conflicto que tenemos, porque hablamos en términos completamente diferentes. (Entrevista 1)

Otro tipo de conflicto entre aseguradoras y prestadores se da, según algunos usuarios, por la falta de pagos de las aseguradoras a las prestadoras, lo que compromete la garantía del acceso de los afiliados a la prestación de servicios. Así lo comenta una usuaria de Golden Group en Ibagué:

\footnotetext{
Ahorita no hay ni urgencias [...] que día pregunte [...] ¿qué urgencias habían abiertas?, me dijeron "no hay sino el Federico Lleras", [pero] el Federico Lleras está en huelga. Entonces ¿A dónde va a ir la gente? No sé en estos días si habrán arreglado con alguna clínica, pero hasta la semana pasada no habían arreglado [...] porque ahí hay más clínicas,
}

\begin{abstract}
entidades, pero ellos no habían organizado nada porque debían. Entonces eso es horrible que uno vaya a sacar una cita y digan "No, no la atendemos porque su EPS nos debe no sé cuántos millones". Y ya uno gastar una plata como gasté yo para ese examen, 30 mil pesos se fueron en droga, y llegar uno y decirle "No, no le hago el examen porque no ha pagado su EPS". (Entrevista 33)
\end{abstract}

Conflictos con el Estado. Los agentes también aluden a conflictos entre el Estado y las aseguradoras en salud. Para el gerente de un hospital público en Cali, cuando el Estado delega en terceros la responsabilidad del aseguramiento, el interés público se desconfigura porque surge un conflicto entre la salud como una responsabilidad del Estado y la salud como un medio para fines privados (Entrevista 24). Un experto en salud también describe conflictos entre el Estado y los gremios, recordando reuniones en las que si "[...] el Ministro empieza a [...] regular de alguna forma el tema de medicamentos, se convierte en un blanco [de] los gremios, porque allí hay unos intereses económicos [...] y cuando hay plata de por medio" surgen esos conflictos (Entrevista 36).

\section{Prima el interés financiero sobre el goce efec-} tivo del derecho a la salud. Un gran número de actores siente que en el sistema de salud prevalece el interés financiero de algunos agentes, spor encima de la salud. Algunos directivos de hospitales públicos y privados indicaron que las reglas de juego se han construido de tal manera que prima el criterio financiero (entrevistas 1 y 30), otros consideraron que, al concentrarse en vigilar aspectos financieros, el sistema descuida la vigilancia de la atención en salud. En ese sentido, el gerente de una clínica privada en Ibagué menciona que "[...] no estamos vigilando lo que debería ser, es decir [...] si a este señor le hicieron su examen odontológico, si le hicieron su control de hipertensión, 
de diabetes, y si tiene una atención adecuada desde el punto de vista primario de salud. Y nos dedicamos es a perseguir dinero. Esa sería mi primera percepción de que el Sistema está al revés, está volcado [...]” (Entrevista 12). De forma similar, la presidenta de la asociación de usuarios de una aseguradora en Calima observó: "La única preocupación que yo veo es a dónde vamos a meter la plata, aquí no se ha hablado de mejorar nada más, [...] [se preguntan] [...] quién maneja la plata, no quien va a solucionar el problema de la negligencia en la salud de los colombianos y la prestación" (Entrevista 2).

\section{Crisis regulatoria generalizada}

Para esta categoría se presentan los testimonios relacionados con los códigos denominados fallas regulatorias, información incompleta, inconsistencia temporal e instituciones y prácticas regulatorias para la toma de decisiones.

Fallas regulatorias. Un experto constitucional adujo que al construir el modelo de salud sobre la marcha y de forma experimental, se produjo un exceso de normas que generan incertidumbre y reglas confusas o poco claras, lo cual complica el papel de los entes de control. En ese contexto, el experto reconoció una falta de capacidad de rectoría, pero insistió en que "[...] antes de eso hay una polución normativa que hace que entender cómo fluye eso [sea] como hacer física cuántica, es muy difícil” (Entrevista 15).

Por otra parte, el presidente de una aseguradora explicó la persistencia de las fallas regulatorias por la falta de actualización y discusión de mecanismos regulatorios como el POS, de acuerdo con las necesidades de los afiliados en el tiempo. Estas circunstancias han producido un aumento desmedido de tutelas a manos de ciudadanos que buscan proteger su derecho a la salud; por ejemplo, cuando la aseguradora les niega los pañales.
Si esto se quiere corregir y si se quiere trascender la idea de las aseguradoras como violadoras del derecho a la salud, es necesario crear los mecanismos para que los pañales ya no se nieguen, incluyéndolos en el POS, si así lo está demandando la población, y haciendo los ajustes correspondientes en la Unidad de Pago por Capitación (UPC) ${ }^{9}$. (Entrevista 28).

Información incompleta. La información incompleta es entendida por distintos actores como un problema estructural y una falla regulatoria del sistema. El superintendente de Salud de turno y un miembro de la Junta Directiva de la Asociación Colombiana de Empresas de Medicina Integral (Acemi) ${ }^{10}$, reconocen que los sistemas de información actuales son débiles y desarticulados, y resaltan que la vigilancia no se realiza con base en información actualizada, ya que esta no se encuentra disponible, lo que genera ineficiencias dentro del sistema. Al respecto, el integrante de la Junta Directiva de Acemi expresó:

[...] [se] necesita un sistema único de información [en salud]. Yo le he insistido al gobierno que debería haber un chasis [con] la información básica [...] de todos los colombianos, y donde se [tengan] los indicadores de salud [conectados] por familias, para saber los riesgos. Y eso tendría que ser el soporte para todo, y cada institución debería construir sobre eso y deberían existir puentes para que cuando, por ejemplo, Enrique consulta a la clínica Shaio, la clínica sepa la información básica. Esa era una función regulatoria del gobierno, el hecho de no tenerla nos pone en un atraso de veinte años. (Entrevista 20)

9 Capitation Payment Unit (CPU). La UPC "es el valor anual que se reconoce por cada uno de los afiliados al sistema general de seguridad social en salud (SGSSS) para cubrir las prestaciones del Plan Obligatorio de Salud (POS), en los regímenes contributivo y subsidiado" (16).

10 Colombian Asociation of Comprehensive Medicine Enterprises (CAIME). 
Según una auxiliar de la Personería ${ }^{11}$ en Calima, la ausencia de un sistema de gestión de información puede obstaculizar el acceso a los servicios, ya que en algunas ocasiones se niega la atención a los usuarios porque los prestadores y las aseguradoras no saben si el ciudadano está o no afiliado al sistema (Entrevista 9). Por lo anterior, la mayoría de los agentes coinciden al proponer que el Gobierno debería esforzarse por promover políticas para garantizar: información de calidad, oportuna y confiable, así como un mayor uso de ella en la IVC, para la garantía del GED.

Inconsistencia temporal. La inconsistencia temporal en la regulación es vista por algunos actores como el resultado de un gran volumen de normatividad, que responde a temas particulares e intereses de los funcionarios del momento, porque como lo manifestó el superintendente de Salud de turno, “[...] cuando llega un ministro interpretan una cosa, cuando llega otro van sacando otras normas [...]" (Entrevista 22). Para el presidente de la Junta Directiva de Acemi, lo anterior va de la mano con la ausencia de políticas de largo plazo, lo que afecta negativamente a la IVC. En ese sentido, el agente afirma:

Colombia es un país experto en hacer normas, pero no en trazar políticas. Me explico: traza cosas temporales no las deja madurar, no las deja crecer y las mata antes de ejecutarlas [...] ha creado normas para resolver particularidades, no para resolver al interior del Sistema, y eso ha hecho [...] que la IVC, llámese Superintendencia y demás, legisle frente a la norma de turno [...] cuando existía el Consejo Nacional de Seguridad Social en Salud (CNSSS) ${ }^{12}$ y la antigua Comisión de Regulación en Salud (CRES) ${ }^{13}$, ellos sacaron

11 Municipal Ombudsman’s Office (MO).

12 National Council on Social Security in Health (NCSSH).

13 Commission on Health Regulation (CHR).
436 acuerdos el CNSSS, otros 18-30 la CRES.

En 12-14 años de funcionamiento, eso daba una norma cada 15 días, eso no permite regular nada. (Entrevista 32)

\section{Instituciones y prácticas regulatorias para} la toma de decisiones. En lo relativo a la existencia de espacios para la toma de decisiones regulatorias en salud, se identifican tres perspectivas. En primer lugar, diversos directivos de aseguradoras y prestadores de servicios de salud en la capital defendieron la necesidad de espacios de concertación y diálogo entre los distintos actores del sistema, abogando por la recuperación de entidades como el CNSSS (entrevistas 26, 30 y 32). En esa dirección, el gerente de una aseguradora en Bogotá indicó que la Ley Ordinaria de Salud (2014) generó mucha polarización:

[...] los estudiantes marchando, los médicos protestando. Y [...] eso no lleva a nada. Entonces [hay] una falta de cohesión social alrededor del tema de la salud [que debería ser resuelta por] los grupos de interés, o sea, uno debería pensar que el CNSSS debería volver a la mesa de gobierno [porque] ahí está sentado el asegurador, el prestador, el municipio, el departamento, la academia [...] consensuando en una mesa los intereses del colectivo. (Entrevista 26)

De manera similar, un miembro de la junta directiva de Acemi está de acuerdo con que el CNSSS era muy valioso porque permitía la participación de todos los agentes y porque en su interior se daban las discusiones necesarias. No obstante, insiste en que las decisiones deben estar en manos del MSPS. Es decir, que el Ministerio debe "[...] ser autónomo en la toma de decisiones, pero preguntar mucho" (Entrevista 20).

Para la segunda perspectiva, la concertación no es deseable, y debe ser sustituida por un ejercicio puramente consultivo en cabeza del 
MSPS, donde se tenga en cuenta la opinión de los actores, pero el MSPS sea quien tome las decisiones finales. Un funcionario del MSPS planteó que lo que se requiere es un órgano consultivo, mas no instituciones como el CNSSS o la CRES, ya que es ineficiente buscar convocar a escala nacional todas las asociaciones de usuarios, asociaciones científicas y médicas, entre otras, para lograr consensos. En cambio, sugiere que debería formarse un órgano consultivo, con representantes elegidos periódicamente de cada grupo de interés, para la formulación de propuestas y que sea el ministro de Salud quien decida si acoge o no dichas propuestas del órgano (Entrevista 16).

Por último, se encuentra la perspectiva que es crítica con respecto a iniciativas como el CNSSS, porque su dinámica democrática es cuestionable. En este caso, es necesario retomar la propuesta de la CRES, que estaba basada en criterios técnicos para informar las decisiones en salud, y donde dichas decisiones eran independientes del Gobierno. A pesar de sostener este punto de vista, un funcionario del Ministerio de Hacienda y Crédito Público (MHCP) $)^{14}$ también expresa que "la idea [de la CRES] era buena”, pero se dañó al llevarla a la práctica, en los procesos de nominación de los técnicos, porque en muchos casos representaban intereses particulares (Entrevista 25).

\section{Discusión}

\section{La legitimidad en el Sistema de Salud}

De acuerdo con los resultados arrojados por las entrevistas, la corrupción y el manejo indebido de los recursos financieros del sistema se generan porque los agentes, especialmente los privados, privilegian el interés individual.

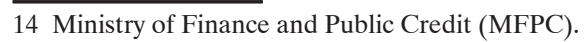

Así las cosas, en lugar de destinar recursos a lo que debe ser, se asignan para inversiones en prestadores o en otros ámbitos de los mercados inmobiliarios, como lo demuestra el caso de SaludCoop. Por esta razón, el dinero no alcanza para garantizar la salud en el sistema, aun cuando los recursos son suficientes. Se entiende entonces que la corrupción, al movilizar el accionar de los actores hacia el bien individual, promueve la atomización de los intereses en el sistema, borrando del mapa la idea del interés general. ManriqueVillanueva y Eslava-Schmalbach apoyan estas ideas, mostrando que "los recursos no han llegado a los usuarios del Sistema debido a la corrupción[...]" y que prevalecen unos valores culturales que promueven la apropiación de recursos de salud, impidiendo que estos sean suficientes para la provisión de servicios (17). De forma similar, Gómez-Arias y Nieto indican que Colombia cuenta actualmente con recursos considerables para la salud, pero gracias a la cadena de intermediación gran parte de este dinero no llega al usuario (6). De esta manera, las aseguradoras han incurrido en el ejercicio sistemático de captura de rentas, a través del desvío de dineros y el fraude, imponiendo altos costos al sistema. Un claro reflejo de esta situación son, según los autores, los recobros injustificados por medicamentos y servicios no incluidos en el POS, que para el año 2010 alcanzaron 5.6 billones de pesos.

$\mathrm{El}$ alto grado de incumplimiento de la numerosa normatividad que existe en el sector salud se entiende como un tema íntimamente ligado a la impunidad que reina en el sistema, todo lo cual puede afectar adversamente los derechos en salud y la legitimidad del sistema. Estudios previos apoyan este argumento, aduciendo que hay una gran diversidad de normas para regular los contratos en el sistema, pero el Gobierno no tiene la capacidad de asegurar su cumplimiento, y son los funcionarios del nivel central los primeros en in- 
cumplir (2). Otro artículo sobre el sistema de información para la calidad en salud insiste en que "los organismos de vigilancia y control no aplican sanciones por incumplimiento en el reporte, lo que favorece la baja adherencia a la norma" (18). Por su parte, Marín y Chávez aportan evidencia de diversas normas vulneradas por aseguradoras y prestadores, lo que incrementa la vulnerabilidad de los pacientes de urgencias (19).

Con nuestro estudio también se encontró que las intenciones de aseguradoras en salud y prestadores de servicios son contradictorias, o como mínimo apuntan en direcciones diferentes, lo que genera un conflicto entre sus intereses. Mientras que las primeras buscan la reducción de costos y la rentabilidad, las segundas quieren asegurar las prestaciones en salud y conservar la vida de los pacientes. La falta de encuentro entre estas visiones se puede tomar como un indicio de la fragmentación en el sistema, a través de un cortocircuito entre los propósitos de aseguradoras y prestadores y frente a los resultados en salud de los usuarios. Esta idea se confirma con el testimonio de un médico, según el cual los gerentes de aseguradoras nunca le preguntan cuáles son sus tasas de infecciones o mortalidad, solamente indagan por el valor de su tarifa (5). La situación también se corrobora en un estudio que explica que ambos actores pertenecen a cadenas de producción distintas, orientadas en direcciones diferentes: una hacia el seguro y la otra hacia las prestaciones médicas (20).

Otro conflicto que surge entre prestadores $y$ aseguradoras en salud tiene que ver con la falta de oportunidad en los pagos de las aseguradoras a los prestadores, situación que compromete la prestación del servicio y el acceso y uso por parte de los afiliados. Estos comportamientos también son una fuente de 46 atomización en el sistema, ya que al retener los recursos en las aseguradoras, se deja de lado y obstaculiza el fin último de la salud, a la vez que el sistema se torna insostenible en términos financieros. Estas circunstancias pueden contribuir a deslegitimar a los actores involucrados y al sistema en su conjunto, alejando al usuario o afiliado. Diversas investigaciones constatan que la lógica rentista de las aseguradoras las lleva a negar y retener el pago de servicios, amenazando el funcionamiento de los prestadores de servicios y el acceso, que queda condicionado a la reputación de la aseguradora como pagadora $(2,5)$. Finalmente, la falta de oportunidad en los pagos genera un "enorme pasivo con las Instituciones Prestadoras de Servicios" (6), lo que denota la amenaza para la estabilidad financiera del sistema.

En relación con conflictos que involucran al Estado, encontramos percepciones según las cuales, por un lado, la delegación del aseguramiento en terceros hace que la salud deje de entenderse como una responsabilidad pública, y por el otro, los gremios tienen el poder para evitar decisiones regulatorias que favorecen el bien general. En consecuencia, la salud pierde relevancia como un tema de interés colectivo y público, ya sea porque se convierte en un medio para fines privados, o porque se imponen los intereses económicos de ciertos grupos. En cualquier caso, la primacía de lo particular evidencia la descomposición de la rectoría dentro del sistema, lo que dificulta la generación de decisiones orientadas a beneficiarlo en su conjunto. La idea de que el Estado ha abandonado sus responsabilidades en salud se apoya en investigaciones que destacan el adelgazamiento del Estado, que diezmó el control estatal, dando paso a la dinámica de oferta y demanda (6). Otros argumentan que las reformas sanitarias, incluida la reducción del Estado, no mejoran el acceso, la calidad ni la equidad en salud, sino que, por el contrario, desestimulan las acciones de prevención y promoción, porque no son 
rentables (5). El poder de los gremios es evidente, dado que la moral rentista del sistema condiciona la toma de decisiones, de tal forma que predominan intereses de grupo y particulares (5). Un ejemplo de esto lo proporciona un estudio según el cual los gremios privados fueron los grandes ganadores del proceso de implementación de la Ley 100, pues tuvieron en el Estado a un aliado protector de sus intereses y divorciado del interés general (21).

Debe señalarse que la retracción del Estado como responsable de la salud de los ciudadanos ha generado un aumento en el recurso a la tutela por parte de los colombianos que buscan asegurar su derecho, lo cual a su vez ha evidenciado un vacío en la capacidad del MSPS como ente regulador del sistema. Frente a este contexto de desprotección del ciudadano e incapacidad del Estado para solventar la situación, y teniendo en cuenta el acervo de tutelas existentes, la CC empezó a asumir una labor de regulación constitucional, por la vía de la Sentencia T-760, entre otras, exigiéndole al MSPS y a la SNS garantizar el GED. El rol de la Corte como regulador constitucional no solo rebasa sus funciones, sino que representa una innovación institucional que refleja la tergiversación de los roles en el interior del sistema, lo que puede provocar inestabilidad.

Siguiendo nuestros resultados, la primacía de lo financiero sobre el GED se impone como una característica fundamental de los conflictos en el seno del sistema colombiano, con reglas trazadas para darle más importancia a ese criterio y una preocupación latente por el manejo de los recursos económicos y la rentabilidad, entre otros, dejando en segundo plano los resultados en salud y el estado de salud de los pacientes. Se fragmenta así el objetivo de la salud como un derecho, y esta queda reducida al carácter de mercancía que deviene en ciertos réditos. En consonancia con estos hallazgos, distintos investigadores reconocen el carácter utilitarista de la Ley 100 (22) y documentan percepciones de agentes que muestran que ya no importan la vida y la salud de los pacientes, sino el dinero $(5,23)$. De manera similar, otros reportan que la búsqueda de rentabilidad por parte de las aseguradoras se da a costa de las obligaciones con el usuario (6).

\section{Crisis regulatoria generalizada}

Entre las fallas regulatorias identificadas por los agentes está el exceso de normas o polución normativa, lo cual genera un paisaje regulatorio muy difícil de entender y fomenta el comportamiento experimental por parte de diversos agentes. Este panorama se ha documentado en distintas investigaciones que reconocen el carácter muchas veces contradictorio de la multiplicidad de normas, lo que genera confusión y dificulta su interpretación y aplicación $(2,18,24)$. Según los entrevistados, las fallas también se encuentran en la falta de actualización de mecanismos regulatorios como el POS, de acuerdo con las necesidades de la población. En esa dirección, Cardona et al. muestran que un gran número de prestaciones exigidas a través de la tutela no fueron incluidas en el POS (25). Los autores también constatan que aun cuando los contenidos del POS2012 evidencian la estructuración de un plan de beneficios más integral y cercano a las necesidades de la población, es preciso asegurar que los prestadores de salud presten estos servicios de forma efectiva y oportuna, lo cual ya se ha visto que puede ser complicado.

Nuestros hallazgos también arrojan que el exceso de normas en salud reproduce un sistema desordenado, donde no existe una consistencia de las reglas en el tiempo que permita solucionar problemas de fondo y en el largo plazo. No es posible contrastar estos 
datos con los de otros estudios en el tema, ya que la literatura sobre inconsistencia temporal de las normas en el sistema colombiano es muy reducida. En consecuencia, es necesario llevar a cabo investigaciones que complementen el tema.

En la misma línea de nuestros resultados, algunos investigadores muestran que el sistema de información en salud colombiano está desarticulado y segmentado $(24,26)$, a la vez que no proporciona datos actualizados, tornándose así en un sistema de baja calidad o ineficiente, donde es urgente agregar datos a nivel central (26). Por otra parte, Marín y Chávez respaldan nuestros hallazgos reportando que la falta de información y su baja calidad o actualización puede generar la negación de los servicios, cuando aseguradoras y prestadores no saben si el usuario está afiliado (19).

Finalmente, en nuestros resultados no hay unanimidad en cuanto al papel de los espacios en los que se deben tomar las decisiones regulatorias: algunos creen que estas debe hacerse desde instituciones como el CNSSS, que promuevan el diálogo y el consenso entre distintos agentes; otros pregonan la necesidad de que se encarguen entidades técnicas autónomas del gobierno, del tipo de la CRES; mientras otros rescatan la pertinencia de la consulta a diversos actores, siempre y cuando la decisión final quede en manos del MSPS. Lo que ha quedado claro en ese contexto es que el tránsito de un tipo de entidad a otra puede causar inestabilidad, a la vez que el predominio de estructuras técnicas o de consulta puede quebrantar los espacios de formación de consensos, que son tan importantes porque permiten el acercamiento de distintos actores del sistema, y como tal están dotados de una legitimidad democrática. Este es un signo más, sumamente claro, de la atomización que experimenta el sistema por la imposibilidad de tejer acuerdos, tomar decisiones conjuntas, conciliar intereses contrapuestos, velar por el bien público y cohesionar las acciones de los agentes en su interior.

En su investigación sobre la rectoría y gobernanza en el sector salud colombiano, Roth-Deubel y Molina Marín reportan que la autoridad sanitaria no logra articular a los agentes del sistema alrededor de objetivos comunes o la formulación de políticas y programas de salud, y, por el contrario, resaltan la imposición de intereses personales sobre intereses colectivos como uno de los desafíos de la rectoría (24). Igualmente, muestran que la gobernanza del sistema exige consolidar espacios de construcción conjunta de decisiones que superen la lógica del ánimo de lucro.

Queda claro que nuestros resultados se corresponden con los de otros estudios científicos sobre percepciones relacionadas con el sistema de salud colombiano, el cual se caracteriza por el dominio de intereses individuales y rentistas sobre el bien general y la garantía del GED. Vale la pena resaltar la importancia de consolidar espacios que permitan la construcción colectiva entre los diversos actores del sistema, como una vía fundamental para la superación de los conflictos y la crisis.

\section{Limitaciones}

Entre las limitaciones de este estudio se debe mencionar que, por ser de tipo cualitativo, los resultados no son generalizables ni representativos, y solo aplican a la población estudiada. Sin embargo, al proporcionar detalle en las percepciones y significados que los entrevistados otorgan a la realidad social, esta investigación aporta un entendimiento preliminar importante en cuanto a los problemas que componen la crisis del sistema de salud colombiano, que pueden resultar de gran relevancia para avanzar en su superación. 


\section{Conclusión}

La combinación de crisis de legitimidad, crisis regulatoria generalizada e incapacidad de las aseguradoras en salud para responder por sus obligaciones, fue generando condiciones percibidas por los agentes públicos y privados como una crisis sectorial que afecta las expectativas futuras para el desarrollo del derecho a la salud y la garantía del goce efectivo al acceso, uso y calidad de los servicios, hasta el punto de que aquellos indagados por si estaban dispuestos a realizar nuevas inversiones, contestaron en su mayoría que no, porque no había condiciones para recuperar en el futuro los recursos invertidos en el presente, y que cumplir con las obligaciones actuales con hospitales y clínicas requería una acción directa del Estado para inyectar nuevos recursos, mejorar el flujo de estos mediante el giro directo e intervenir las entidades que se encontraban al borde de la quiebra por malos manejos y corrupción, como Saludcoop y Caprecom.

La CC fue llenando los vacíos regulatorios mediante órdenes y procesos de seguimiento, lo cual dio origen a la regulación constitucional para la protección del derecho a la salud, y el MSPS fue encargándose de la administración directa de los recursos, y los beneficios, en una especie de seguro público unificado "de hecho", en la medida que a través del giro directo de los recursos el MSPS paga en forma directa las prestaciones de servicios, interviene administrativamente las aseguradoras más grandes y mediante el sistema de reembolso denominado UPC administra los recursos de las aseguradoras.

La regulación corriente, responsabilidad del MSPS, se trasladó a la CC, y la administración de los riesgos y los recursos, responsabilidad de las aseguradoras, se ha ido delegando al MSPS, lo que origina, informalmente, un proceso de integración entre la dirección del sistema, el financiamiento y el aseguramiento, que no resulta muy eficiente con las reglas de operación definidas por la Ley 100. Este sistema, que funciona para administrar la crisis, configura un marco de expectativas pesimistas sobre el futuro de los agentes independientes, una realidad regulatoria inesperada por las nuevas funciones de la CC y un marco de debilitamiento de la mezcla pública y privada. En tales condiciones y según la opinión de los agentes, es necesaria una reforma ordinaria que desarrolle el derecho a la salud como un derecho fundamental y ponga en concordancia el qué de los derechos con los medios existentes en el SGSSS.

\section{Referencias bibliográficas}

1. Ministerio de Salud y Protección Social. Plan Obligatorio de Salud [internet]. [acceso: 19 de marzo del 2017]. Disponible en: https://www.minsalud.gov. co/salud/Paginas/pos.aspx

2. Molina Marín G, Vargas Jaramillo J, Berrío Castaño A, Muñoz Marín DP. Características de la contratación entre aseguradores y prestadores de servicios de salud, Medellín, 2007-2008. Rev Gerenc Polit Salud. 2010;9(18):103-15.

3. Presidencia de la República de Colombia. Decreto 1876 de 1994, por el cual se reglamentan los artículos 96, 97 y 98 del Decreto Ley 1298 de 1994 en lo relacionado con las Empresas Sociales del Estado [internet]. Diario Oficial de la República de Colombia; 1994 [acceso: 9 de marzo del 2017]. Disponible en: http://www.alcaldiabogota.gov.co/ sisjur/normas/Norma1.jsp? $\mathrm{i}=3356$

4. Ministerio de Salud y Protección Social. Aseguramiento al sistema general de salud. Cuáles son las funciones de las entidades promotoras de salud? [internet]. [acceso: 9 de marzo del 2017]. Disponible en: https://www.minsalud.gov.co/proteccionsocial/ Regimensubsidiado/Paginas/aseguramiento-al-sistema-general-salud.aspx

5. Molina G, Ramírez A. Conflicto de valores en el sistema de salud de Colombia: entre la economía de mercado y la normativa constitucional, 2007-2009. Rev Panam Salud Pública [internet]. 2013;33(4):287-93. Disponible en: http://search. ebscohost.com/login. aspx? direct $=$ true $\& \mathrm{db}=0$ fm\&AN $=87528356 \&$ lang $=$ es \&site $=$ ehost-live

6. Gómez-Arias RD, Nieto E. Colombia: ¿Qué ha pasado con su reforma de salud? Rev Peru Med Exp Salud Publica. 2014;31(4):733-9.

7. Mayan M, Cisneros C. Una introducción a los métodos cualitativos: módulo de entrenamiento para 
estudiantes y profesionales. Int Inst Qual Methodol [internet]. 2001;53. Disponible en: http://www. ualberta.ca/ iiqm//pdfs/introduccion.pdf

8. Fresneda O, Moreno P, Alfonso O. La red urbana colombiana: una visión a partir del tamaño funcional y la especialización económica de las ciudades. Santafé de Bogotá: Ministerio de Desarrollo Económico; 1998.

9. Wengraf T. Interview "facts" as evidence to support inferences to eventual theorization / representation models. En: Qualitative research interviewing [internet]. Londres: Sage Publications; 2001. p. 2-16. Disponible en: doi: http://dx.doi. org/10.4135/9781849209717.n1 Print

10. Wellington J, Szczerbinski M. Some qualitative methods considered. En: Research methods for the social sciences. Londres: Continuum International Publishing; 2007. p. 79-99.

11. May T. Social surveys: design to analysis. En: Social research issues, methods and process [internet]. Maidenhead, Berkshire: McGraw Hill, Open University Press; 2001. p. 27. Disponible en: https://www-dawsonera-com.ludwig.lub.lu.se:2443/ readonline/9780335239986/startPage/88

12. Semb O, Kaiser N, Andersson S, Sundbom E. Learning psychology as a challenging process towards development as well as " studies as usual ": a thematic analysis of medical students' reflective writing. Advamces Med Educ Pract. 2014;(5):491-8.

13. Green J, Willis K, Hughes E, Small R, Welch N, Gibbs L, et al. Generating best evidence from qualitative research: The role of data analysis. Aust N Z J Public Health. 2007;31(6):545-50.

14. Vaismoradi M, Turunen H, Bondas T. Content analysis and thematic analysis: Implications for conducting a qualitative descriptive study. Nurs Health Sci [internet]. 2013;(15):398-405. Disponible en: http://doi.wiley.com/10.1111/nhs.12048

15. Mieles Barrera MD, Tonon G, Alvarado Salgado SV. Investigación cualitativa: el análisis temático para el tratamiento de la información desde el enfoque de la fenomenología social. Univ Humanística [internet]. 2012;(74):195-25. Disponible en: http:// revistas.javeriana.edu.co/index.php/univhumanistica/article/view/3648\%5Cnhttp://www.redalyc.org/ articulo.oa? $\mathrm{id}=79125420009$

16. Ministerio de Salud y Protección Social. Unidad de Pago por Capitación del Régimen Subsidiado [internet] [acceso: 19 de marzo del 2017]. Disponible en: https:// www.minsalud.gov.co/salud/Paginas/UPC_S.aspx)
17. Manrique Villanueva L, Eslava Schmalbach J, Sc M. Auscultando la corrupción en la salud: definición y causas. ¿Qué está en juego? Rev Colomb Obs Ginecol. 2011;62(4):308-14.

18. Restrepo Sierra LH, López Ríos MM. Sistema de información para la calidad en salud : una realidad por explorar, para conocer y decidir responsablemente. Rev CES Salud Pública. 2015;6(1):54-64.

19. Marín O YA, Chávez G BM. Inconsistencias en la afiliación al sistema de salud colombiano: barrera administrativa para la atención integral de las urgencias médicas. Rev Fac Nac Salud Pública [internet]. 2014;32(1):62-70. Disponible en: http://search.ebscohost.com/login.aspx?direc$\mathrm{t}=$ true $\& \mathrm{db}=\mathrm{a} 9 \mathrm{~h} \& \mathrm{AN}=94336348 \&$ lang $=$ es \& site $=$ ehost-live

20. Restrepo JH, Lopera JF, Rodríguez SM. La integración vertical en el sistema de salud colombiano. Rev Econ Inst. 2007;9(17):279-308.

21. Uribe Gómez M. La dinámica de los actores en la implementación de la reforma del sistema de salud en Colombia: exclusiones y resistencias 1995-2003. Rev Gerenc Polit Salud. 2009;8(17):44-68.

22. Escobar J. Ley 100 de seguridad social: implicaciones bioéticas. En: Escobar J, Da Costa M, Jácome S, Málaga H, Maldonado C, Montt J, et al., editores. Bioética y justicia sanitaria, segunda edición. Bogotá: El Bosque; 1999.

23. Echeverry López M. Reforma a la salud y reconfiguración de la trayectoria de acceso a los servicios de salud desde la experiencia de los usuarios en Medellín, Colombia. Rev Gerenc Polit Salud. 2011;10(20):97-109.

24. Roth-Deubel A, Molina-Marín G. Rectoría y gobernanza en salud pública en el contexto del sistema de salud colombiano, 2012-2013. Rev Salud Pública [internet]. 2013;15(1):44-55. Disponible en: http:// www.scielosp.org/pdf/rsap/v15n1/v15n1a05.pdf

25. Cardona Á, Mejía LM, Vargas GS, Álvarez E, Nieto E. Análisis de la actualización del Plan Obligatorio de Salud del Sistema de Seguridad Social en Salud colombiano, 2009-2012. Rev Salud Pública [internet]. 2013;15(2):258-70. Disponible en: http://search.ebscohost.com/login.aspx?direct $=$ true $\& \mathrm{db}=1$ th $\& \mathrm{AN}=95644905 \&$ lang $=$ es $\&$ site $=$ eds-live

26. Bernal Acevedo O, Forero Camacho JC. Sistemas de información en el sector salud en Colombia. Rev Gerenc y Políticas Salud. 2011;10(21):85-100. 